\title{
Factors affecting utilization of Insecticide treated nets among people living with HIVIAIDs in Bahir Dar city, northwest Ethiopia
}

\author{
Yibeltal Berie $^{1}$, Kasahun Alemu ${ }^{2}$, Alemayehu Belay ${ }^{3}$, Zemichale Gizaw ${ }^{4}$ \\ ${ }^{1}$ Department of Health promotion and disease prevention Core Process, Amhara National Regional Health Bureau, Bahir Dar City, Ethiopia \\ ${ }^{2}$ Institute of Public Health, College of Medicine and Health Sciences, Gondar University, Gondar City, Ethiopia \\ ${ }^{3}$ Department of Health Education and Promotion, Felege Hiwot Referral Hospital, Bahir Dar City, Ethiopia \\ ${ }^{4}$ Department of Environmental health Institute of Public Health, College of Medicine and Health Sciences, Gondar University, Gondar City, \\ Ethiopia
}

\section{Email address:}

yiberie@gmail.com(Y.Berie),alemukass@yahoo.com(K.Alemu),balemayehu95@yahoo.com(A. Belay), zemichael12@gmail.com(Z.Gizaw)

\section{To cite this article:}

Yibeltal Berie, Kasahun Alemu, Alemayehu Belay, Zemichale Gizaw. Factors Affecting Utilization of Insecticide Treated Nets among People Living with HIV/Aids in Bahir Dar City, Northwest Ethiopia. Science Journal of Clinical Medicine.

Vol. 2, No. 6, 2013, pp. 147-152. doi: 10.11648/j.sjcm.20130206.11

\begin{abstract}
Introduction: Malaria and HIV are among the two most important global health problems of this time. All HIV-positive persons (PLHAs) in the household were encouraged to sleep under insecticide treated nets (ITNs) at all times of year. However, the information on utilization and associated factors on these groups have been limited. Objective: the aim of the study was to assess utilization of ITN and associated factors among PLHA, who are members of the three PLHA associations in Bahir City Administration, northwest Ethiopia. Methods: A community based cross-sectional study was conducted from March to May, 2013. A simple random sampling technique was used to select the study participants. Pre-tested and structured questionnaire and observation check list was used to collect the data. Each variable were analyzed by using bi variate logistic regression to know their significance and to assess the separate effects and multi variate analysis was done using backward stepwise logistic regression to control the possible confounding effect for variables with p-value $<0.2$ in bi variate analysis. Result: Three hundred four304 (76.8\%) of PLHA utilized ITNs properly. The main reasons for those who had not have ITN and not utilized ITN were, they did not know where obtained the ITNs $20(41.7 \%)$, ITNs were expensive $16(33.3 \%)$, and no fear of mosquito bite at this time 60(65.2\%). Knowledge about malaria risks and ITNs importance $(\mathrm{AOR}=2.3 ; 95 \%$ CI 1.23- 4.40), formal education $(\mathrm{AOR}=2.39 ; 95 \%$ CI 1.40- 4.08) and better income was significantly associated with ITN utilizations ( $\mathrm{AOR}=1.83 ; 95 \% \mathrm{CI} 1.05-3.20)$ Conclusion: The PLHA needs special attention on the utilization of ITN. Free provision of ITNs, awareness creation on the utilization of ITN and participate in Income generating activities are supreme important.
\end{abstract}

Keywords: Malaria Control, PLHAS, ITNS Utilization, Bahir Dar City, Ethiopia

\section{Introduction}

Malaria and HIV are among the two most important global health problems of this time. Malaria in combination with HIV cause more than 4 million deaths a year and are both concentrated primarily in Sub-Saharan Africa, Asia and South America. Furthermore, more than 500million cases of malaria occur every year and at least a million of them cause deaths. An estimated 30-36million people are living with $\mathrm{HIV}$ in Africa, resulting in more than 3 million deaths In
Ethiopia, malaria is one of the most important public health problems, with more than three-quarters of the landmass (altitude $<2000 \mathrm{~m}$ ) of the country is either malariaous or potentially malariaous, and an estimated $68 \%$ ( $>50$ million people) of the total population resides in areas at risk of malaria infections(1, 2). In 2009/2010, malaria was the leading cause of outpatient visits and health facility admissions, accounting for $14 \%$ of outpatient visits and $9 \%$ of admissions $(3,4)$.

In 2010, the Ethiopia Federal Ministry of Health $(\mathrm{EFMOH})$ reported 4,068,764 clinical and confirmed 
malaria cases to the World Health Organization (WHO) (5).Use of ITNs is one of the major vector control measures in Ethiopia. More than 20 million ITNs were distributed between 2005 and 2007, enabling $68 \%$ of the households living in malaria-endemic areas to own at least one ITN (6-8). Pregnant women, children under five years of age and PLHA are at considerably higher risk of contracting malaria and suffering from or dying of it than others (9).

Results from the malaria indictor survey 2007 reflected that significant effort of the $\mathrm{FMOH}$, scale-up malaria prevention and control interventions with substantial increases in ITN ownership and use as well as malaria knowledge. The 2011 malaria indictor survey didn't show an improvement in LLIN ownership or use compared with 2007 malaria indictor survey but those seeking treatment within 24 hours and women's malaria knowledge were markedly improved(10).

All HIV-positive persons in the household were encouraged to sleep under ITNs at all times of year, however, the information on utilization and associated factors for these groups have been limited. Hence, this study aimed to identify the utilization of ITN and associated factors among PLHA. The finding would be helpful for policy makers and managers and malaria prevention and control partners to insight their plans.

\section{Methods}

A community based cross sectional study design was used to assess utilization of ITN and associated factors among PLHA, who were members of the three associations with atotal of 2227 PLHAs (Tesfagohe=932, Mekidem Ethiopia=1201 and Wogagen=94)in Bahir City Administration, from March to May 2013which is the capital city of Amhara National Regional State and $565 \mathrm{Km}$ far from Addis Abeba. It is located at $11^{\circ} 38^{\prime}$ latitude and at $37^{\circ} 10^{\prime}$ East longitudes at $1801 \mathrm{~m}$ above sea level. Bahir Dar is one of the tourist destinations in the country and strongly affected by Malaria and HIV/AIDS epidemics.

\subsection{Sampling Procedure}

The study included all PLHAs who were members of the three PLHA associations, and age greater than 18 years old. Simple random sampling technique was used to select the participants and random number was generated to increase unpredictability of the selection. A total of 396 PLHA were included in the study.

participants who answered at least six and above series of knowledge assessing questions related to malaria and ITN were considered as sufficiently knowledgeable.ITN utilization was considered when presence of bed nets were hanged over the bed and participants slept in the previous night under the nets.

\subsection{Data Collection and Analysis}

The pretested and structured questionnaires were used to collect the data. The questionnaires were prepared in English and translated in to the local language (Amharic) and then back-translated into English to check for any inconsistencies or distortions in the meaning of words and concepts and to increase the validity of the data. The questionnaire comprised socio demography, behavioral and economical factor data's. Observation check list was used to confirm the utilization of ITNs.

The data were entered, cleaned and edited using in Epi Info version 3.5.3 and exported to SPSS version20.0 software for further analysis. Analysis was made by using bi variate and multi variate logistic regression. Each variable were analyzed by using bi variate logistic regression to know their significance and to assess the separate effects and multi variate analysis was done using backward stepwise logistic regression to control the possible confounding effect for variables with $\mathrm{p}$-value $<0.2$ in bi variate analysis. P-value of less than 0.05 was considered as statistical significant. Odds ratio (OR) with $95 \%$ confidence interval was used to assess the presence and degree of association between dependent and independent variables.

\subsection{Ethical Consideration}

Ethical clearance was obtained from University of Gondar ethical review committee; both written and verbal permissions were secured from Regional Health Bureau, Bahir Dar city Administration health office, Bahir Dar city HAPCO and NAP+ Bahir Dar office. Then official letter were written to each respective PLHA associations. Informed consent was obtained from the participants before conducting data collection. All the study participants informed about the objective and importance of the study and were also informed about their right of not participating in the study at any time. Privacy and confidentiality of the information was assured and collected anonymously.

\section{Results}

\subsection{Socio-Demographic Characteristics of the Study Population}

Three hundred eighty eight (98.01\%) participants were participated in the study. The mean age of respondents was $35.68 \pm 8.54$ years. About $74 \%$ (293) were female and $44.7 \%$ (177) were married. About 31.8\% (126) were attended formal education from grade 1 to 8 and $27.5 \%$ (109) of the respondents were illiterate. Regarding the occupational status of the participants, government and private employees were the highest $34.8 \%$ (138) followed by daily laborers $26 \%$ (103), merchants $24.6 \%$ (97), and housewife $6.8 \%$ (27), and drivers $4 \%$ (16). The majority 97\% (384) were orthodox in religion. The income distribution reveals that the poorest/lowest quintile (from the respondent's level) accounted for $32.1 \%$. (Table 1 ) 
Table 1: Socio demographic characteristics of the respondents in Bahir Dar city administration, northwest Ethiopia, May 2013 (n=396)

\begin{tabular}{|c|c|c|c|}
\hline Characteristics & & frequency & $\begin{array}{l}\text { Percent } \\
(\%)\end{array}$ \\
\hline \multirow{4}{*}{ Age } & $18-24$ & 23 & 5.8 \\
\hline & $25-44$ & 316 & 79.8 \\
\hline & $45-64$ & 54 & 13.6 \\
\hline & $>64$ & 3 & 0.8 \\
\hline \multirow{2}{*}{ Sex } & Male & 103 & 26 \\
\hline & Female & 293 & 74 \\
\hline \multirow{5}{*}{ Marital status } & Married & 177 & 44.7 \\
\hline & Single & 49 & 12.4 \\
\hline & Divorced & 95 & 24 \\
\hline & Widowed & 66 & 16.7 \\
\hline & Separated & 9 & 2.3 \\
\hline \multirow{5}{*}{$\begin{array}{l}\text { Educational } \\
\text { status }\end{array}$} & Illiterate & 109 & 27.5 \\
\hline & $\begin{array}{l}\text { Can read and } \\
\text { write }\end{array}$ & 86 & 21.7 \\
\hline & 1-8 Grade & 126 & 31.8 \\
\hline & 9-12 Grade & 68 & 17.2 \\
\hline & $12+$ & 7 & 1.8 \\
\hline \multirow{6}{*}{$\begin{array}{l}\text { Occupational } \\
\text { status }\end{array}$} & Driver & 16 & 4 \\
\hline & $\begin{array}{l}\text { Gove./private } \\
\text { employees }\end{array}$ & 138 & 34.8 \\
\hline & Merchant & 97 & 24.6 \\
\hline & Daily laborer & 103 & 26 \\
\hline & House wife & 27 & 6.8 \\
\hline & Others & 15 & 3.8 \\
\hline
\end{tabular}

\begin{tabular}{llll}
\hline Characteristics & frequency & $\begin{array}{l}\text { Percent } \\
(\%)\end{array}$ \\
\hline \multirow{2}{*}{ Religion } & Orthodox & 384 & 97 \\
& Muslim & 7 & 1.8 \\
& Protestant & 5 & 1.2 \\
& $>\$ 16$ & 127 & 32.1 \\
& $\$ 16-21.6$ & 54 & 13.6 \\
Income & $\$ 21.6-27$ & 63 & 15.9 \\
& $\$ 27-37.8$ & 92 & 23.2 \\
& $>\$ 37.8$ & 60 & 15.2 \\
$\begin{array}{l}\text { Number of } \\
\text { families }\end{array}$ & $>3$ & 269 & 67.9 \\
\hline
\end{tabular}

\subsection{Knowledge and ITNs Utilization}

The results revealed that the majority of the respondents, $348(87.9 \%)$ had ITNs and $304(76.8 \%)$ of them utilized ITNs properly. The majority $339(85.6 \%)$ were sufficiently knowledgeable about malaria and ITNs. About 304 (76.8\%) of respondents heard information from Health professionals and $57(14.4 \%)$ were from mass medias. Of those had an ITNs, the majority $261(75 \%)$ had one ITN. The major source of the nets, 327 (93.9\%) were free provision by the government and malaria prevention and control partners. All family members, 228(57.6\%) and 65 (16.4\%) PLHAs slept under the bed nets prior night to the survey day. Lack of information where to obtain ITNs $20(41.7 \%)$, lack of money to purchase it $16(33.3 \%)$ and lack of awareness on its usage $7(14.6 \%)$ were the main reasons for unavailability of ITNs in their home (Table 2).

Table 2: Availability and status of ITN and knowledge of the respondents in Bahir Dar city administration, northwest Ethiopia, May 2013. (n=396)

\begin{tabular}{|c|c|c|c|}
\hline Characteristics & & frequency & Percent (\%) \\
\hline \multirow{2}{*}{ Knowledge about malaria and ITNs } & sufficiently knowledgeable & 339 & 85.6 \\
\hline & Not sufficiently knowledgeable & 57 & 14.4 \\
\hline \multirow{3}{*}{ Source of information } & Health professionals & 304 & 76.8 \\
\hline & Media & 57 & 14.4 \\
\hline & Community & 35 & 8.8 \\
\hline \multirow{3}{*}{ Availability of ITN } & One & 261 & 75 \\
\hline & Two & 79 & 22.7 \\
\hline & Three & 8 & 2.3 \\
\hline \multirow{2}{*}{ Source of ITN } & Buying & 21 & 6.1 \\
\hline & Freely from government/NGo & 327 & 93.9 \\
\hline \multirow{4}{*}{ Sleep under ITN prior to survey day } & All family & 228 & 57.6 \\
\hline & PLHAs & 65 & 16.4 \\
\hline & $<5$ years children & 22 & 5.6 \\
\hline & Nets are expensive & 16 & 33.3 \\
\hline \multirow{3}{*}{ Reasons not having ITNs } & Not know the source where they got & 20 & 41.7 \\
\hline & Nets have not any use & 7 & 14.6 \\
\hline & Others & 5 & 10.4 \\
\hline
\end{tabular}


The main reason that they were not using ITNs was no fear of mosquito bite at this time $60(65.2 \%)$, ITN caused thermal stress $31(33.7 \%)$ and took time every night 19(20.7\%). (Fig2)

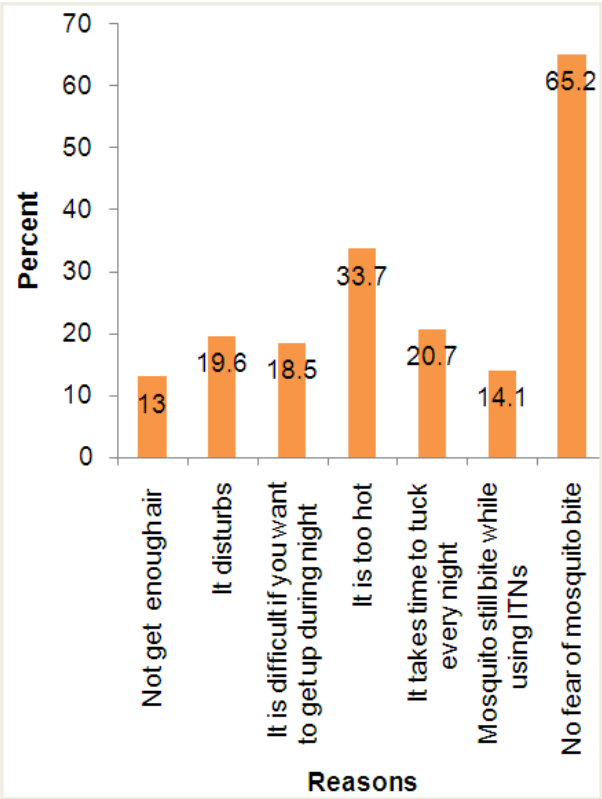

\subsection{Associated Factors for ITN Utilization}

Among the potential associated factors explored regarding utilization of ITNs among PLHAs, having sufficient knowledge about transmission of malaria and ITNs, educated formal education (primary to university level), employed in government/private organization, had better income were significant predictors.

The respondents those who have sufficient knowledge about malaria and ITNs were 2.3 times more likely to utilize ITN than those who have insufficient knowledge about malaria and ITNs $(\mathrm{AOR}=2.3 ; 95 \%$ CI 1.23-4.40). In the study educated formal education (primary to university level) were 2.4 times more likely to utilize ITN than those not educated formal education ( $\mathrm{AOR}=2.39 ; 95 \% \mathrm{CI} 1.40-4.08)$, beside this those who have better income were 1.8 times more likely utilize ITN than have less income $(\mathrm{AOR}=1.83$; 95\%CI1.05-3.20) and also those are employed in government/private organization were 3 times less likely utilize ITN than non employees $(\mathrm{AOR}=0.33 ; 95 \% \mathrm{CI}$ 0.19-0.57)(Table 3).

Fig 2: Respondents reasons not using ITN in Bahir Dar city administration May, 2013

Table 3: Variables associated with ITNs utilization in Bahir Dar city administration, northwest Ethiopia, May 2013.

\begin{tabular}{|c|c|c|c|c|c|c|}
\hline \multicolumn{2}{|l|}{ Variables $(n=396)$} & \multicolumn{2}{|c|}{$\begin{array}{l}\text { Utilization of } \\
\text { ITN }\end{array}$} & $\operatorname{COR}(95 \% \mathrm{CI})$ & $\operatorname{AOR}(95 \% C I)$ & p-value \\
\hline \multirow[b]{2}{*}{ knowledge status } & $\begin{array}{l}\text { Have Sufficient } \\
\text { Knowledge }\end{array}$ & 267 & 72 & $1.85(1.07,3.19)^{*}$ & $2.32(1.23,4.40)^{*}$ & 0.01 \\
\hline & Not have sufficient Knowledge & 37 & 20 & 1 & & \\
\hline \multirow{2}{*}{$\begin{array}{l}\text { Educational } \\
\text { status }\end{array}$} & Not have formal education & 136 & 59 & 1 & & \\
\hline & Have formal education & 168 & 33 & $5.10(3.51,7.39)^{*}$ & $2.39(1.40,4.08)^{*}$ & 0.001 \\
\hline \multirow{2}{*}{$\begin{array}{l}\text { Occupational } \\
\text { status }\end{array}$} & Non employees & 201 & 47 & 1 & & \\
\hline & Employees & 103 & 45 & $0.54(0.32,0.88)^{*}$ & $0.331(0.19,0.57)^{*}$ & $<0.001$ \\
\hline \multirow{2}{*}{ Income } & Below mean(500) & 177 & 67 & 1 & & \\
\hline & Above mean & 127 & 25 & $5.08(3.31,7.80)^{*}$ & $1.829(1.05,3.20)^{*}$ & 0.034 \\
\hline \multirow{2}{*}{$\begin{array}{l}\text { Number of } \\
\text { families }\end{array}$} & $<3$ & 212 & 57 & $3.72(2.78,4.98)^{*}$ & - & \\
\hline & $>\mathbf{3}$ & 92 & 35 & 1 & - & \\
\hline \multirow{2}{*}{ Age } & $<45$ & 274 & 77 & $1.78(0.91,3.48)$ & - & \\
\hline & $>45$ & 30 & 15 & 1 & & \\
\hline \multirow[b]{2}{*}{ Sex } & Male & 76 & 27 & 1 & - & \\
\hline & Female & 228 & 65 & $1.25(0.74,2.10)$ & - & \\
\hline \multirow[b]{2}{*}{ Marital status } & With couples & 136 & 41 & $1.01(0.63,1.61)$ & - & \\
\hline & Without couples & 168 & 51 & 1 & - & \\
\hline \multirow{2}{*}{ Source of ITNs } & Buying & 17 & 4 & $1.73(0.55,5.41)$ & - & \\
\hline & Freely from GO/NGO & 278 & 39 & 1 & - & \\
\hline
\end{tabular}

* Statistically significant at $\mathrm{p}<0.05$ 


\section{Discussion}

In this study the overall utilization of ITNs were low. Respondents did not know the source of ITNs and nets are expensive are the main reasons that did not had ITN and also no fear of mosquito bite at this time, ITN caused thermal stress, took time every night and it caused disturbance are the reasons that the respondents did not use ITNs. Among the potential determinants explored regarding utilization of ITNs having sufficient knowledge about malaria and ITNs, educated formal education (primary to university), non employees with in government/private organization and have higher income are significant predictors of ITNs utilization.

In this study the overall coverage of ITN were high this is much more than another study conducted from three sites in Kenya 34.5\% reported owning an optimal bed net (11), this is probably because of greatest effort taken by $\mathrm{FMOH}$, HAPCO and PLHAs association to prevent malaria. Even though it is higher coverage, considering the respondents need special attention, the study area are urban \&malariaous, to achieve the $6^{\text {th }}$ MDGs and use of bed net are the best and east intervention methods to prevent malaria than other intervention packages the coverage is low.

The result revealed that the utilization of ITNs were $76.8 \%$ this is almost similar to other studies conducted from three sites in Kenya (Kilifi, Kisii, and Kisumu)76.9\%(11), but much less than another studies conducted in Rakai, Uganda $91 \%$,this is because in Uganda ITN utilization were followed by a special program. Like ITN coverage considering the respondents need special attention, the study area are urban \&malariaous, to achieve the $6^{\text {th }}$ MDGs and use of bed net are the best and east intervention methods to prevent malaria than other intervention packages the utilization is very low.

As a result the reason not having ITNs are respondents did not know the source of ITNs and nets are expensive this is similar to another studies conducted in Nigeria lack of money to buy the commodity and did not know where to buy the net. This is not surprising because majority of the PLHA are not have formal education and have no means of livelihood. Although the ITNs are distributed freely, it is still impossible for them to replace the nets those are out of use because of lack of money (12).The reason that they were not using ITNs was it is too hot/heat this is also similar to another studies conducted in Nigeria (12) and Rakai, Uganda (13).Even though not described in other studies no fear of mosquito bite at this time, took time every night, it caused disturbance, it is difficult if you want to get up during night, mosquito still bite while using ITNs and there is no enough air are the main reason that they were not using ITNs this is because most of the respondents are not have formal education, low income and have information about burden of malaria are decreased.

Among the potential determinants explored regarding utilization of ITNs PLHAs who are a member of three
PLHAs association in Bahir Dar city administration, having sufficient knowledge about malaria and ITNs, educated formal education (primary to university), non employees with in government/private organization and have higher income are significant predictors of ITNs utilization. The respondents those who have sufficient knowledge about malaria and ITNs were 2.3 times more likely to utilize ITN than those who have insufficient knowledge about malaria and ITNs(AOR=2.3; 95\% CI 1.23-4.40) this is because if people know about malaria infection and the prevention methods they use ITN to protect from malaria infections. In the study educated formal education (primary to university level) were 2.4 times more likely to utilize ITN than those not educated formal education $(\mathrm{AOR}=2.39$; $95 \% \mathrm{CI} 1.40$ 4.08) this is similar to another studies conducted from three sites in Kenya (Kilifi, Kisii,and Kisumu) this is because if people education formal education they know the impact of malaria and their prevention methods beside this those who have better income were 1.8 times more likely utilize ITN than have less income $(\mathrm{AOR}=1.83 ; 95 \% \mathrm{CI} 1.05-3.20)$ this is similar to another studies conducted from three sites in Kenya (Kilifi, Kisii, and Kisumu) this is because people can buy and replace their bed nets and also those are employed in government/private organization were 3 times less likely utilize ITN than non employees $(\mathrm{AOR}=0.33 ; 95 \% \mathrm{CI}$ $0.19-0.57)$ this is similar to another studies conducted from three sites in Kenya (Kilifi, Kisii,and Kisumu) this is because even they are employed in government or private organization the majorities are not have formal education and employed as cleaners, grads and store keepers to motivate the PLHAs.

Findings suggest that lower socioeconomic status, not have sufficient knowledge about malaria and ITN, not educated formal education and have permanent job are a barrier to proper utilization of bed net.

\subsection{Strengths of the Study}

The study focused on the most vulnerable segment of the population. Early morning survey was carried out to observe the actual behavior of the respondents with regard to ITNs utilization. The non-response rate was almost none even during the early morning survey

\subsection{Limitation of the Study}

The study not includes all PLHAs live in Bahir Dar city administration. Character of HIV status (CD4 Count and WHO stage) not included in the study.

\section{Conclusions}

The study declared that the coverage and utilization of ITN is low. Reason not having ITNs is respondents did not know the source of ITNs and nets are expensive to replace and also the main reason that they were not using ITNs properly was no fear of mosquito bite at this time, it is too 
hot and it takes time to tuck every night. Education, knowledge, income and occupation are significant predictors of ITNs utilization. Findings suggest that lower socioeconomic status, not have sufficient knowledge about malaria and ITN, not educated formal education and have permanent job are a barrier to proper utilization of bed net.

\section{Recommendation}

Free distribution of ITNs will be provided by the Bahir Dar city administration Health office and other partners' working around this area. Re-orientation of the staff of the PLHAs association to increase commitment and effectiveness in the control of malaria (using ITNs) by NAP+ and HAPCO (Bahr Dar office) is highly recommended. Intensive behavioral change communication carried out by association workers and volunteers to remove barriers on utilization of ITNs. Enhance training by NAP+ and HAPCO (B/Dar office) for volunteers' and association leaders towards proper utilization of ITNs.

\section{Author's Contribution}

YB conceptualized the research problem, designed the study, conducted field work, collected data, and drafted the manuscript.

KA was involved in preparing the research proposal, research report, and revision of the manuscript.

AB was involved in revision of the research proposal, data analysis and revision of the manuscript for publication.

ZG was involved in revision of the proposal and research report

All authors of the manuscript have read and agreed to its content.

\section{Acknowledgements}

The authors are grateful University of Gondar and Amhara regional Health Bureau for providing scholarship. We would like to thank United State Agency for International Development (USAID) MSH/ENHAT- CS program for financial support. We would like to appreciate PLHA associations in Bahir Dar city administration, data collectors and supervisors and the participants.

\section{References}

[1] Adhanom T DW, Witten KH, Getachew A, Seboxa T. Malaria. In: Berhane Y, Haile-Mariam, D., Kloos, H (Eds), editor. Epidemiology and Ecology of Health and Disease in Ethiopia. Addis Ababa: Shama Books; 2006. p. 556-76.

[2] WHO. Malaria and HIV/AIDs interaction and implication; conclustion of technical consulation. 2004.

[3] Health FDRoEMo. Integrated Management of Newborn and Childhood Illness. Addis Ababa. FMOH; 2008.

[4] Health FDRoEMo. Health and Health Related Indicators, Addis Ababa: FMOH, 2009/2010.

[5] World Malaria Report : www.who.int/ malaria/ world_malaria_report_2011/en/.[27/02/2013].

[6] Eisele TP LD, Steketee RW. Protective efficacy of interventions for preventing malaria mortality in children in plasmodium falciparum endemic areas. Int $\mathrm{J}$ Epidemiol. 2010;39:i88-i101.

[7] FMOH. National Malaria Guideline. Addis Ababa: Federal Minitry of Health of Ethiopia; 2012.

[8] WHO. Insecticide-Treated Mosquito Nets: a WHO Position Statement. . Geneva: World Health Organization; 2007.

[9] WHO U. Protecting vulnerable groups in malaria-endemic areas of Africa through accelerated deployment of insecticide-treated nets. WHO and UNICEF. 2005:1-2.

[10] CDC U. Presidant Malaria initative, Ethiopia Malaria Operational Plan. 2013.

[11] Obinna N. Nnedu GCJ-S, Benson O. Singa , Ben Piper , Phelgona A. Otieno, Alicia Guidryg, Barbra A. Richardson and Judd Walson Prevalence and correlates of insecticide-treated bednet use among HIV-1-infected adults. AIDS Care 2012; 24(12):1559_64.

[12] Patience Edoho Samson-Akpan OBE, Ekaette Francis Asuquo, Mary Achi Mgbekem and Idang Neji Ojong. control of malaria among PLWHA in Calabar,crossRiver State, Nigeria: Life science; 2012.

[13] Lauren Cohee LAM, Joseph Kagaayi, Ilana Jacobs, Ronald Galiwango, James Ludigo, Joseph Ssekasanvu and Steven J Reynolds. High retention and appropriate use of insecticide-treated nets distributed to HIV-affected households in Rakai, Uganda: results from interviews and home visits. Malaria Journa. 2009; 8 (76). 